\title{
Técnica de embalsamiento de cadáver según Thiel. Un método valioso para entrenar y mejorar las destrezas quirúrgicas en el campo de la otorrinolaringología
}

\author{
Thiel embalming technique for cadáver preservation: An overview of a valuable \\ method for training and improvement of the surgical skills in Otolaryngology
}

\author{
Jhonder Salazar G',2, Antonio Arroyo S $^{2}$, Juan Gras A², Francisco Sánchez D².
}

\begin{abstract}
RESUMEN
Los tiempos modernos nos obligan a que la formación de un médico cirujano no se base sólo en conocimientos teóricos, sino que adquiera las mayores habilidades quirúrgicas para así evitar riesgos innecesarios en una sociedad altamente exigente. La disección y el entrenamiento de los procedimientos quirúrgicos juegan un papel primordial para cumplir este objetivo, por lo que recientemente se ha promovido una forma de preservación cadavérica que permite realizar mediante situaciones bastantes realistas dichos adiestramientos. Se realizó una búsqueda bibliográfica electrónica, restringida al idioma inglés en Pubmed, Scopus y WOS donde se pretende describir y analizar las aplicaciones de esta técnica. La revisión ofreció artículos de relevancia que demuestran la aplicación de esta técnica novedosa en la preservación de cadáveres y sus posibles aplicaciones en el campo de otorrinolaringología.
\end{abstract}

Palabras clave: Método de embalsamamiento Thiel, entrenamiento quirúrgico, otorrinolaringología.

\begin{abstract}
Nowadays, it is not possible to train a healthcare professional only based on theoretical knowledge, but acquiring the highest surgical skills to avoid unnecessary risks in a highly demanding society, therefore dissection and training of surgical procedures play a key role in fulfilling this objective, which is why a form of cadaveric preservation has recently been promoted, which allows realizing such training through realistic situations. An electronic literature search was carried out, restricted to the English language in MEDLINE, where it is intended to describe and analyze the applications of this technique. The review offered articles of relevance that demonstrate the application of this novel technique in the preservation of corpses and their possible applications in the field of otorhinolaryngology.
\end{abstract}

Key words: Thiel embalming method, surgical training, otolaryngology.

\footnotetext{
Servicio de Otorrinolaringología, Hospital Universitario Vall d’Hebron, Barcelona, España.

2 Programa de Doctorado en Salud Pública, Ciencias Médicas y Quirúrgicas. Universidad Miguel Hernández de Elche. Alicante, España.
} 


\section{INTRODUCCIÓN}

El alto nivel de exigencia en la atención y calidad técnica de procedimientos quirúrgicos, junto con la necesidad de un alto conocimiento anatómico, han exigido recientemente a los médicos cirujanos un grado de excelencia y maestría sin precedentes. El dominio quirúrgico requiere de la combinación de conocimientos y habilidades manuales, por lo que tradicionalmente la cirugía se ha enseñado con el aprendiz operando a pacientes bajo la supervisión de un tutor; sin embargo, hay factores como la disminución en los tiempos de entrenamiento quirúrgico por la presión médico-asistencial, el alto riesgo de procedimientos invasivos realizados por manos inexpertas, los recientes cambios en las directrices europeas en relación al tiempo para entrenar cirujanos, la alta resolución de casos complejos en hospitales no universitarios, entre otros, que han hecho que se tenga que buscar alternativas mediante el uso de diversos modelos de entrenamiento para enseñar, reforzar y mejorar las destrezas técnicas en la práctica quirúrgica'

En general los especialistas quirúrgicos siguen buscando modelos que les permitan simular los aspectos prácticos de sus cirugías y evitar las complicaciones propias de la exposición in vivo, en especial en aquellas donde se requiere de alta precisión quirúrgica, por lo que han ensayado diferentes modelos que incluyen simuladores virtuales en ordenadores, modelos sintéticos inanimados, animales vivos o cadáveres frescos o preservados. A través de los programas de realidad virtual se pueden obtener conocimientos básicos en el autoaprendizaje de forma relativamente barata pero con el inconveniente de carecer de las relaciones anatómicas o de la manipulación real de tejidos, así como de la falta de resistencia o retroalimentación de las fuerzas aplicadas sobre ellos que se obtienen en los modelos reales tan necesarios particularmente en la cirugía, por lo que en general estos programas se consideran como un primer paso en el entrenamiento quirúrgico ${ }^{1}$.

Los modelos sintéticos de plástico, por ejemplo de material compuesto, no proveen del realismo ni de sensación al tacto similar a la del vivo, pero pueden fabricarse en grandes cantidades y usarse múltiples veces, por lo que se aceptan como una primera aproximación de acceso ilimitado, y por tanto relativamente barata para el aprendizaje. Hay destrezas o habilidades técnicas que pueden adquirirse en modelos sintéticos de baja fidelidad con el mismo grado de beneficio que el entrenamiento en un programa de alta fidelidad, un ejemplo de esto puede ser la sutura o la eliminación endoscópica de una piedra ureteral, sin embargo, hay otros donde la fidelidad anatómica y del tejido juegan un gran papel y no pueden ser sustituidos².

Los modelos de animales vivos proveen una sensación realista comparable a las de un entorno clínico, pero cuyas estructuras anatómicas no son obviamente las mismas que en seres humanos, además del hecho que los procedimientos deben ser realizados en animales anestesiados (requiriendo de personal entrenado para tal fin) y que finalmente se sacrifican, lo que genera el rechazo de buena parte de la comunidad pública sin menoscabo de las trabas legales en cuanto a legislaciones de cada país y los costos que todo el proceso genera ${ }^{3}$.

Los cadáveres frescos, pese a ser los más usados presentan muchos problemas que limitan su uso, entre ellos, la cinética de la rigidez cadavérica y la putrefacción que solo permite usarlos por un periodo de tiempo muy corto, aumentando el riesgo de exposición a agentes microbianos como virus o bacterias. Para acortar la brecha entre la muerte y el uso técnico de cadáveres en entornos educativos, en especial en el área del entrenamiento quirúrgico, es necesario preservarlos teniendo en cuenta que la elección en el método de conservación afecta en gran medida su uso futuro; se considera que la preservación es apropiada cuando el cadáver se mantiene a salvo de daños, destrucción o descomposiciones. Esto se consigue tratando el cadáver con productos químicos especiales, es decir, embalsamándolos, proceso que tiene como objetivo preservar y desinfectar el cuerpo humano indefinidamente ${ }^{3,4}$.

Durante muchos años uno de los productos químicos más importantes utilizados para este propósito ha sido el formaldehído, basado principalmente en la formalina. Con la expansión de las necesidades de entrenamiento quirúrgico, se observó que este tipo de preservación carecía de cualidades para el manejo adecuado debido a los cambios significativos que presenta en la fuerza, color, fragilidad de los órganos y tejidos, es decir, 
en los cadáveres preservados mediante formaldehído, la anatomía es real pero la sensación al tacto y la recreación de la cirugía no lo es debido a la rigidez del cadáver ${ }^{4}$.

Un aspecto importante a considerar en la práctica de conservación y disección de cadáveres es el efecto nocivo del formaldehído sobre la salud humana. Actualmente existen normas que regulan su uso y manejo en la mayoría de los países y desde el año 2006 la Agencia Internacional para la Investigación sobre el Cáncer (IARC) lo clasifica como cancerígeno para seres humanos ${ }^{3}$.

Por todo esto, los cuerpos embalsamados tienen la ventaja de llevar un riesgo mínimo de infección y ser adecuados para un uso prolongado. Los procedimientos se hacen normalmente en un cuerpo completo, proporcionando una experiencia realista. Sin embargo, dependiendo del tipo de embalsamamiento, pueden ocurrir cambios en la movilidad, el color, separación de los planos o la dureza y manejo del tejido 5 .

Gracias a las técnicas de embalsamamiento, el uso de cadáveres está en aumento, ya que no sólo se han empleado clásicamente en el aprendizaje de la anatomía sino que también juegan un papel importante como modelo quirúrgico en situaciones en las que no resulte factible, legal 0 ético trabajar directamente con pacientes. Son ampliamente utilizados para el entrenamiento en especialidades quirúrgicas con la ventaja de que permiten a los cirujanos cometer errores o probar nuevos enfoques y/o abordajes técnicos. También los cadáveres contribuyen al desarrollo preclínico de los instrumentos y procedimientos utilizados en la cirugía, permitiendo a los equipos de diseño probar nuevos dispositivos o técnicas e identificar áreas de mejora en las primeras etapas de desarrollo del producto. Los ingenieros y diseñadores de productos tienen la oportunidad de probar sus productos de una manera práctica, algo que no es posible con un paciente real.

El objetivo de esta revisión es conocer el uso de cadáveres embalsamados mediante la técnica de Thiel en formación quirúrgica de especialistas en otorrinolaringología, en sesiones de entrenamiento de procedimientos quirúrgicos de especialistas relacionadas con el campo otorrinolaringológico y determinar cómo se percibe su uso como herramienta fidedigna de entrenamiento y aprendizaje anatómico. Este artículo se basa en una revisión narrativa en los buscadores Pubmed, Scopus y WOD con las estrategias de búsqueda [(otolaryngology OR surgical training) AND (thiel embalming)] para identificar los estudios primarios que evaluaron el uso de cadáveres embalsamados mediante la técnica de Thiel en el entrenamiento quirúrgico del área otorrinolaringológica. Se incluyeron revisiones, ensayos clínicos, artículos en inglés y español de 2007-2017. Se excluyeron abstracts a congresos, casos clínicos, aplicación en otras áreas 0 especialidades médicas (Figura 1). Se seleccionaron 22 artículos que cum-

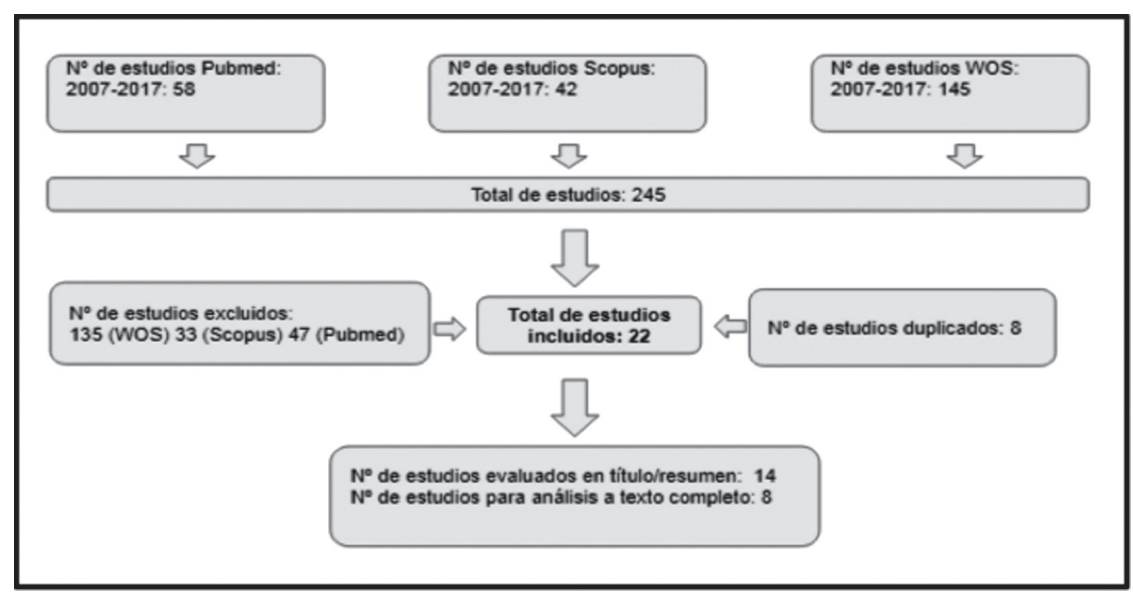

Figura 1. Método de selección de artículos. 
plieron los criterios de inclusión. Se descartaron artículos de otras áreas de especialización o que no se relacionan con procedimientos en el área otorrinolaringológica (ver Tabla 1).

\section{MÉTODO DE EMBALSAMAMIENTO SEGÚN THIEL ¿EN QUÉ CONSISTE?}

Es una técnica desarrollada en el Departamento de Anatomía de Graz (Austria) por el anatomista Walter Thiel en 1992, quien desde principios de los años 60 intentó buscar una alternativa en la conservación de cadáveres que no resultase tóxica ni carcinógena como el formol, y adicionalmente permitiera que los cuerpos mantuvieran la flexibilidad y apariencia de un cuerpo vivo $0^{6,7}$.

Durante los primeros años, luego de su publicación, fueron pocos los centros anatómicos que comenzaron a usarla, especialmente germanoparlantes, debido en parte a la pobre difusión de esta técnica (publicada inicialmente en alemán), sus altos costos (aproximadamente $300 £$, en comparación con unos $30 £$ utilizando un método más clásico, basado en formaldehído) y las adaptaciones infraestructurales que deben realizarse en el departamento anatómico para llevarla a cabo, hace evidente que se necesiten recursos financieros considerables para permitir su uso rutinario especialmente en países en vía de desarrollo ${ }^{8}$.

Este embalsamamiento ha sido diseñado y refinado en las últimas tres décadas, se trata de un fluido a base de agua, glicol, varias sales y cantidades muy bajas de formaldehído volátil y formalina para efectuar la fijación del tejido. Toda la mezcla a base de agua consiste en monopropilenglicol, nitrato de amonio, nitrato de potasio, sulfito de sodio, ácido bórico, clorocresol, formalina, y, en el caso de los fluidos de perfusión, también alcohol y morfolina. La alta concentración de componentes salinos en la solución causa una desnaturalización de las proteínas, cuya precipitación conduce a una homogeneización del tejido, manteniéndose la textura fisiológica del mismo. Las bajas cantidades de formaldehído hacen que los cadáveres con esta técnica aporten seguridad para trabajar en ellos, además de ventajas contrastadas con otras técnicas de embalsamamiento ${ }^{9}$. El primer paso del proceso de embalsamamiento según Thiel es la perfusión, que tiene lugar poco después de la llegada del cadáver al departamento de anatomía. Dos líquidos se infunden simultáneamente, uno arterial (normalmente a través de la arteria femoral 0 braquial) y uno venoso (normalmente a través del seno sagital superior o la vena braquial). Después de esto, los cuerpos se sumergen en un tanque

Tabla 1. Estudios para análisis a texto completo con sus principales resultados

\begin{tabular}{|c|c|c|c|c|}
\hline Autor y col. & Año & Tipo de estudio & $\mathrm{n}$ & Resultado principal \\
\hline Benkhadra & 2009 & Aleatorizado & 16 & Fidelidad de la anatomía del cuello \\
\hline Szücs Z & 2016 & Observacional & 16 & Anatomía de la vía aerodigestiva superior \\
\hline Eisma & 2011 & Comparativo & 5 & Entrenamiento en cirugía tiroidea \\
\hline Stieger & 2012 & Experimental & 23 & Mecanismo del oído medio, membrana timpánica, estribo, ventana redonda. \\
\hline Guignard & 2013 & $\begin{array}{l}\text { Observacional } \\
\text { Experimental }\end{array}$ & 7 & $\begin{array}{l}\text { Desplazamiento de la base de cráneo, umbo, } \\
\text { promontorio coclear, estribo, ventana oval }\end{array}$ \\
\hline Feigl & 2008 & $\begin{array}{l}\text { Observacional } \\
\text { Experimental }\end{array}$ & 96 & Entrenamiento del abordaje transmeatal del nervio ampular posterior \\
\hline Havel & 2014 & $\begin{array}{l}\text { Observacional } \\
\text { Experimental }\end{array}$ & 1 & $\begin{array}{l}\text { Contribución de la cavidad nasal y paranasal } \\
\text { en las propiedades resonadoras del tracto vocal }\end{array}$ \\
\hline Gras-Cabrerizo & 2014 & Prospectivo & 2 & $\begin{array}{l}\text { Entrenamiento y diseño anatómico de colgajos } \\
\text { pediculados procedentes de la arteria esfenopalatina }\end{array}$ \\
\hline
\end{tabular}


con el líquido de embalsamar durante al menos 4-6 meses.

A continuación, pueden ser almacenados en una bolsa de plástico sellada hasta su uso, sin necesidad de refrigeración. También se ha usado esta mezcla para revivir especímenes completos o parciales fijados con formaldehído, proporcionando una alternativa adecuada para institutos que no pueden permitirse todo el proceso completo ${ }^{10}$. El método de embalsamamiento de Thiel preserva durante mucho más tiempo el cadáver con una fidelidad del color, textura, plasticidad y flexibilidad musculoarticular similares a la del espécimen en fresco pero sin la variedad de problemas que incluyen la necesidad de tener congeladores para su almacenamiento y un tiempo de trabajo limitado a escasas horas debido a la rápida putrefacción luego de la descongelación y el riesgo infeccioso que esto conlleva, la explicación de la fidelidad de estas características pudiera ser debido a la integridad de la estructura de colágeno que preserva las fibras musculares y tendinosas ${ }^{9,11,12}$.

La idoneidad de los cadáveres puede mejorarse mediante la inyección de silicona de color (mpolímero) en las grandes arterias, venas, 0 ambos. La perfusión entra en los vasos pequeños, incluso hasta $1 \mathrm{~mm}$ de diámetro, dependiendo de la presión, volumen de inyección y fluidez de la solución de silicona, lo que permite identificar e investigar la irrigación sanguínea de ciertas regiones corporales ${ }^{10,11}$. Los cadáveres pueden retenerse durante muchos años sin necesidad de refrigeración, con poco mantenimiento, sin representar mayores riesgos para la salud de quienes los manipulan y pudiendo permanecer sellados en bolsas de plástico para cadáveres o sumergidos sin la emanación de gases nocivos o irritantes.

Los cadáveres embalsamados con el método Thiel no tienen olor detectable, pero sí una flexibilidad real de las partes del cuerpo, excelente preservación del color del músculo, vísceras y vasculatura, además de propiedades superiores de conservación antimicrobiana y antifúngica que permiten incluso de forma segura diseccionar sin guantes, por lo que han demostrado ser adecuados no sólo para el conocimiento anatómico sino también para el aprendizaje de una amplia gama de procedimientos quirúrgicos. En general, alguna de las principales desventajas que se describen en el uso de este método corresponde a factores intrínsecos propios del donante ante cualquier método de preservación, como serían su edad promedio o condiciones médicas asociadas, que conllevan alteraciones anatómicas 0 dificultades técnicas. La preservación del cerebro es limitada, e implica una manipulación muy frágil aunque presenta su estructura bien conservada en los estudios de resonancia magnética. El cartílago se suaviza con el tiempo y el músculo liso, como en el útero, puede ser bastante suave. La vasculatura con esta técnica de conservación se aplana, pudiendo ser rellenada con material más resistente para evitar su colapso. Así mismo, pudieran presentar problemas con algunas formas de equipos electroquirúrgicos debido a la conductividad de los fluidos de embalsamamiento, o ser inadecuada para investigaciones histológicas.

\section{USO EN DIFERENTES ESPECIALIDADES}

La flexibilidad de estos cadáveres los hace adecuados para procedimientos muy variados, así lo muestran estudios donde se usaron 30 cadáveres preservados con esta técnica para diseñar colgajos libres microvascularizados y documentaron fotográficamente la conservación de las finas estructuras vasculares destacando las condiciones realistas de los vasos y nervios de los colgajos en los que podían incluso semanas después llevar a cabo microsuturas ${ }^{13}$. Se han usado para el aprendizaje de la técnica de colocación de implantes dentales y otras cirugías orales con amplia aceptación por la similitud en la mucosa, hueso y estructuras nerviosas del hueso maxilar ${ }^{14}$. En la cirugía de la columna vertebral se mantiene la alineación y las deformaciones características lo que ha permitido ensayar nuevos abordajes y reparaciones quirúrgicas, además por mantener las características internas de la articulación y flexibilidad son aprovechables en una amplia gama de procedimientos endoscópicos articulares ${ }^{15}$. La posibilidad de trabajar dentro de la cavidad abdominal insuflada ha permitido la manipulación en el interior de estas cavidades y su amplio uso en la cirugía urológica, donde se ha identificado el rol potencial en las áreas de laparoscopía, pieloureterografía retrógrada, endoscopía del tracto 
urinario superior e inferior, angiografía renal, esofagectomía video asistida ${ }^{16-19}$.

También se han utilizado para la angiografía, radiografía intervencionista y otros procedimientos vasculares, incluyendo reparaciones microvasculares. Son ideales para probar y afinar nuevos abordajes quirúrgicos, como cuando se han usado para realizar procedimientos asistidos con láser mínimamente invasivos y así poder recanalizar arterias femorales crónicamente ocluidas ${ }^{20} 0$ evaluar la factibilidad de la mediastinoscopía en la disección linfática ganglionar subaórtica y traqueobronquial mediante nuevas rutas de acceso ${ }^{21}$.

\section{APLICABILIDAD DEL MÉTODO DE THIEL EN OTORRINOLARINGOLOGÍA}

A pesar de las claras ventajas de esta técnica de embalsamamiento de cadáver, en el campo de la otorrinolaringología sus aplicaciones son pocas. Se describe la comparación de técnicas anestésicas en el campo cervical, demostrando la fidelidad de estos modelos para mostrar la anatomía del cuello mejor preservada que en los cadáveres frescos ${ }^{22,23}$. En base a este estudio se comparó el entrenamiento en la cirugía tiroidea en cadáveres con formalina evaluando la calidad del tejido, la percepción al realizar el procedimiento, identificación de estructuras anatómicas, olor y otros concluyendo que el modelo embalsamado es más realista ${ }^{24}$. La flexibilidad del cuello y la apertura oral es normal, lo que hace extrapolar su uso en entrenamiento de cirugía de vía aérea superior, ya que la anatomía y la exposición laríngea están plenamente conservadas ${ }^{23}$.

La microcirugía endolaríngea representa una situación especialmente difícil y delicada que ha sido abordada históricamente mediante modelos de entrenamiento con animales, para los cuales las laringes porcinas se han estudiado desde un punto de vista fonatorio, histológico e inmunohistoquímico, reconociendo su uso extendido por su fidelidad y facilidad de obtención ${ }^{25}$. Sin embargo, según nuestros conocimientos, no se reporta el uso de cadáveres embalsamados con Thiel en la investigación de la endolaringe, reparación de cuerdas vocales, insuficiencia glótica, resecciones o nuevos abordajes mediante el uso láser, entre otros.
Las cabezas humanas embalsamadas según Thiel se pueden utilizar como un modelo alternativo para el estudio de la mecánica del oído medio secundaria a la estimulación a través de conducción ósea, ya que salvo algunas frecuencias donde se hallan diferencias con sujetos vivos, la movilidad de los huesecillos con respecto al promontorio es idéntica, así mismo la preservación de las estructuras del oído interno y del órgano vestibular estimulan a la investigación traslacional basada en los hallazgos intracocleares y vestibulares ${ }^{26,27}$. En ese mismo campo de la otología, se ha ensayado escasamente ciertos abordajes quirúrgicos, como el de neurectomía, demostrándose el papel crucial de la disección de un gran número de especímenes en la mejora de la experiencia del cirujano y tasa de éxito ${ }^{28}$.

Así mismo las propiedades resonadoras y la anatomía de las fosas y senos paranasales se han evaluado en estos cadáveres tras realizar selectivamente cirugía de los ostiums de drenajes y levantamiento de colgajos pediculados intranasales en estudios anatómicos que abre el campo a la amplia variedad de procedimientos endoscópicos nasales y de base de cráneo que pueden realizarse en ellos 29,30 .

\section{CONCLUSIÓN}

Considerando que existen muchas habilidades quirúrgicas que no pueden lograrse practicando directamente sobre el paciente, hay un consenso general sobre la utilización de cadáveres para poner en prácticas dichas habilidades. Los cadáveres con Thiel resuelven los problemas tradicionalmente asociados a la simulación cadavérica, tales como olor perturbante, restricciones éticas y coste. Con todo esto, y reconociendo que los modelos humanos embalsamados son superiores a cualquier otro modelo animal o sintético para el correcto aprendizaje de técnicas y habilidades quirúrgicas creemos que el método de Thiel tiene un gran papel en la formación y aprendizaje de técnicas quirúrgicas del otorrinolaringólogo de nuevos tiempos. Sería de interés para la educación quirúrgica y los entrenamientos en otorrinolaringología evaluar la calidad de los cadáveres Thiel en cirugía laríngea, modelos robóticos, cirugía endoscópica nasal y otológica, entre otros. 


\section{BIBLIOGRAFÍA}

1. Gilbody J, Prasthofer A, Ho K, Costa M. The use and effectiveness of cadaveric workshops in higher surgical training: a systematic review. Ann Roy Coll Surg 2011; 93: 347-52.

2. Groscurth P, Eggli P, Kapfhammer J, Rager G, HORnUng J, FASEL J. Gross anatomy in the surgical curriculum in Switzerland: Improved cadaver preservation, anatomical models, and course development. Anat Rec 2001; 265: 254-6.

3. Fonseca-Matheus J. Conservación de piezas anatómicas para la enseñanza en carreras médicas. Gac Cs Vet 2012; 17: 5-10.

4. Brenner E. Human body preservation - old and new techniques. J Anat 2014; 224: 316-44.

5. EISMA R, WiLkINSON T. From "Silent Teachers" to Models. PLOS Biol 2014; 12: e1001971.

6. THIEL W. An arterial substance for subsequent injection during the preservation of the whole corpse. Ann Anat 1992; 174: 197-200.

7. THIEL W. The preservation of the whole corpse with natural color. Ann Anat 1992; 174: 185-95.

8. Benkhadra M, Gérard J, Genelot D, Trouilloud P, Girard C, Anderhuber F et al. Is Thiel's embalming method widely known? A world survey about its use. Surg Radiol Anat 2010; 33: 359-63.

9. Jaung R, Cook P, BLyth P. A comparison of embalming fluids for use in surgical workshops. Clin Anat 2011; 24: 155-61.

10. Hunter A, Eisma R, Lamb C. Thiel embalming fluid-A new way to revive formalin-fixed cadaveric specimens. Clin Anat 2014; 27: 853-5.

11. Eisma R, Lamb $C$, Soames R. From formalin to thiel embalming: What changes? One anatomy department's experiences. Clin Anat 2013; 26: 564-71.

12. Benkhadra M, Bouchot A, Gérard J, Genelot D, Trouilloud P, Martin L et al. Flexibility of Thiel's embalmed cadavers: the explanation is probably in the muscles. Surg Radiol Anat 2010; 33: 3658.

13. Wolff K, Kesting M, Mücke T, Rau A, Hölzle F. Thiel embalming technique: A valuable method for microvascular exercise and teaching of flap raising. Microsurg 2008; 28: 273-8.

14. Hölzle F, Franz E, Lehmbrock J, Weihe S, Teistra C, Deppe H et AL. Thiel Embalming Technique: A Valuable Method for Teaching Oral Surgery and
Implantology. Clin Implant Dent $R$ 2009; 14 : 121-6.

15. Wilke H, Werner K, Häussler K, Reinehr M, Böckers T. Thiel-fixation preserves the non-linear loaddeformation characteristic of spinal motion segments, but increases their flexibility. $J$ Mech Behav Biomed 2011; 4: 2133-7.

16. Healy S, Ral B, Biyani C, Eisma R, Soames R, $N_{A B I} G$. Thiel Embalming Method for Cadaver Preservation: A Review of New Training Model for Urologic Skills Training. Urology 2015; 85: 499-504.

17. Giger U, Frésard I, Häfliger A, Bergmann M, KRÄHENBÜHL L. Laparoscopic training on Thiel human cadavers: a model to teach advanced laparoscopic procedures. Surg Endosc 2008; 22: 901-6.

18. Lara C, García L, Diez M, Oller I, Aguilar M, Arroyo A, Calpena R, Bellón M. Esofaguectomía videoasistida combinando modelo real con modelo conservación en Thiel. Cir Esp 2016; 94: 999.

19. García L, Molla P, Bellón M, Aguilar M, Guiner L, Galindo I, Arroyo A, Calpena R. Cadáveres Thiel: ¿el futuro en la formación sobre modelos anatómicos? Encuesta de satisfacción. Cir Esp 2016; 94: 778.

20. Heneweer C, Siggelkow M, Helle M, Petzina R, Wulff A, Schaefer J et al. Laser scoop desobliteration: a method for minimally invasive remote recanalization of chronically occluded superficial femoral arteries. Journal of Biomedical Optics 2015; 20: 025005.

21. Tokairin Y, Nagal K, Fujiwara $\mathrm{H}, \mathrm{Og}_{\mathrm{g}} \mathrm{T}$, OKuda M, Nakaulma Yet al. Mediastinoscopic Subaortic and Tracheobronchial Lymph Node Dissection With a New Cervico-Hiatal Crossover Approach in Thiel-Embalmed Cadavers. Int Surg 2015; 100: 580-8.

22. Benkhadra M, Faust A, Ladolre S, Trost 0, Trouilloud P, Girard $C$ et al. Comparison of fresh and Thiel's embalmed cadavers according to the suitability for ultrasound-guided regional anesthesia of the cervical region. Surg Radiol Anat 2009; 31: 531-5.

23. Szücs Z, László C, Baksa G, László I, Varga M, Szuák A et Al. Suitability of a preserved human cadaver model for the simulation of facemask ventilation, direct laryngoscopy and tracheal 
intubation: a laboratory investigation. Brit $J$ Anaesth 2016; 116: 417-22.

24. Eisma R, Mahendran S, Majumdar S, Smith D, SoAmes R. A comparison of Thiel and formalin embalmed cadavers for thyroid surgery training. J Roy Coll Surg Edin 2011; 9: 142-6.

25. Nasser Kotby M, Wahba H, Kamal E, ElMakhzangy A, Bahaa N. Animal model for training and improvement of the surgical skills in endolaryngeal microsurgery. $J$ Voice 2012; 26: $351-7$.

26. Stieger C, Candreia C, Kompis M, Herrmann G, Pfiffner F, Widmer D et al. Laser Doppler Vibrometric Assessment of Middle Ear Motion in Thiel-Embalmed Heads. Otol Neurotol 2012; 33: $311-8$
27. Guignard J, Stieger C, Kompis M, Caversaccio M, Arnold A. Bone conduction in Thiel-embalmed cadaver heads. Hear Res 2013; 306: 115-22.

28. Feigl G, Kos I, Anderhuber F, Guyot J, Fasel J. Development of surgical skill with singular neurectomy using human cadaveric temporal bones. Ann Anat 2008; 190: 316-23.

29. Havel M. Resonator properties of paranasal sinuses: preliminary results of an anatomical study. Rhinology J 2014; 52: 172-82.

30. Gras-Cabrerizo J, Gras-Albert J, Monjas-Canovas I, García-Garrigós E, Montserrat-Gili J, Sánchez del Campo F et al. Colgajos pediculados procedentes de la arteria esfenopalatina: estudio anatómico y quirúrgico. Acta Otorrinolaringol Esp 2014; 65: 242-8. 